\title{
Manifestações renais na síndrome de Joubert
}

\author{
Renal symptoms in the Joubert syndrome
}

\section{Ana Paula Weiss ${ }^{1}$, Maria Cristina de Andrade ${ }^{2}$, João Tomas de A. Carvalhaes ${ }^{3}$}

\section{RESUMO}

Objetivo: Descrever o caso clínico de paciente com Síndrome de Joubert associada a alterações renais.

Descrição do caso: Paciente de dois meses de idade admitida com quadro hipotonia e hiperpneia. Ao exame físico, observaram-se, além da respiração irregular, movimentos oculares anormais e hipertensão arterial; não se evidenciaram alterações na ausculta cardíaca e pulmonar. Durante investigação clínico-laboratorial inicial, as causas cardíacas e pulmonares foram descartadas. Aventaram-se hipóteses diagnósticas de patologias neurológicas com doença renal. Os exames laboratoriais mostraram presença de alcalose respiratória, acidose metabólica e hipercalemia, com função renal normal. A ressonância magnética evidenciou alterações neurológicas compatíveis com "sinal do dente molar", quadro frequentemente associada à Sindrome de Joubert. Levando-se em consideração a associação dessa síndrome com alterações renais, a investigação nefrológica demonstrou imagens císticas em parênquima renal.

Comentários: Patologias cardíacas e pulmonares estão frequentemente associadas a manifestações clínicas como taquipneia e distúrbios metabólicos. Entretanto, pode ser necessária uma investigação neurológica porque diversas doenças que acometem o sistema nervoso central apresentam tais alterações. A associação entre alterações renais e malformações de sistema nervoso central é frequente em diversos processos sindrômicos, justificando-se a sua investigação. A Síndrome de Joubert e as desordens a ela relacionadas caracterizam-se por aplasia do vermix cerebelar, ataxia, movimentos oculares anormais, respiração irregular e retardo do desenvolvimento neuropsicomotor. As alterações renais mais comuns são os cistos renais e a nefronoftise, que pode progredir para doença renal terminal.

Palavras-chave: insuficiência renal; hipotonia muscular; manifestações oculares; ataxia.

\section{ABSTRACT}

Objective: To describe the case of a patient with Joubert syndrome associated with renal impairments.

Case description: A 2 month-old patient was admitted with hypotonia and hyperpneia. At the physical exam, besides irregular breathing pattern, abnormal eye movements and arterial hypertension without abnormalities in cardiac or pulmonary sounds were observed. At the initial clinical and laboratorial investigations, cardiac and pulmonary causes were excluded. The diagnostic hypothesis was: neurological illness associated with renal disease. Laboratorial analysis showed respiratory alkalosis, metabolic acidosis and hyperkalemia, with normal renal function. In the magnetic resonance, images of neurological alterations were compatible with the "molar tooth sign", frequently associated with Joubert syndrome. Renal investigation was performed and cystic images in renal parenchyma were found.

Comments: Cardiac and pulmonary illness are frequently associated with clinical manifestations such as tachypnea and metabolic alterations. Nevertheless, neurological investigation may be necessary, since some diseases that affect the central nervous system may manifest these signs and symptoms. Association between renal alterations and central nervous system malformations are frequent in several diseases and should be investigated. Joubert syndrome and its associated disorders are characterized by aplasia of the cerebellar vermis, ataxia, abnormal eye movements and irregular breathing pattern with psychomotor and mental delay. The most frequent renal problems associated with the disease are renal cysts and nephronophtisis that can progress to end-stage renal failure.

Key-words: renal insufficiency; muscle hypotonia; eye manifestations; ataxia.

Endereço para correspondência

Maria Cristina de Andrade

Rua Gaivota, 754, apto. 71 - Moema

CEP 04522-032 - São Paulo/SP

E-mail: andrademc@globo.com

Recebido em: 8/4/08

Aceito em: 8/9/08 


\section{Introdução}

A Síndrome de Joubert, bem como as desordens a ela relacionadas, caracteriza-se por alterações do sistema nervoso central, oculares e renais ${ }^{(1-3)}$. A principal alteração do sistema nervoso central é a hipoplasia de vérmis cerebelar acompanhada de malformações complexas, que se manifestam clinicamente por hiperpneia e respiração irregular, retardo do desenvolvimento neuropsicomotor, ataxia, hipotonia e, radiologicamente, pelo clássico "sinal do dente molar" ${ }^{(4-10)}$. O principal sinal oftalmológico é a movimentação ocular anormal, sendo frequente a distrofia retiniana e o coloboma ${ }^{(11-13)}$. Apesar de pouco frequentes, as alterações renais mais comuns são os cistos renais e a nefronoftise, podendo haver progressão da doença renal com perda importante da função renal ${ }^{(1)}$.

A taquipneia frequentemente reflete doenças de acometimento pulmonar ou cardíaco. Entretanto, na investigação diagnóstica sempre se deve excluir as alterações do sistema nervoso central, que podem se expressar dessa maneira, principalmente nos casos em que há evidência de alteração do exame neurológico, como a presença de retardo do desenvolvimento neuropsicomotor. Nesse contexto, o objetivo do presente artigo é relatar o caso de uma criança que se apresentou com taquipneia e cujo diagnóstico, após extensa investigação, foi de síndrome de Joubert, além de fazer uma breve revisão da literatura a respeito dessa entidade clínica.

\section{Descrição do caso}

Terceira filha de pais não consanguíneos, nascida de parto normal com 38 semanas de idade gestacional, sexo feminino, peso ao nascer de $3910 \mathrm{~g}$, considerada grande para a idade gestacional, $53,5 \mathrm{~cm}$ de comprimento e $36 \mathrm{~cm}$ de perímetro cefálico. Apresentou boletim de Apgar de 8 e 9 , no $1^{\circ}$ e $5^{\circ}$ minutos de vida, respectivamente. Em ultrassonografia obstétrica realizada na $35^{\text {a }}$ semana gestacional, foi evidenciada hidrocefalia leve. Nos antecedentes familiares, apresentava um irmão falecido com três dias de vida por causa desconhecida.

No segundo dia de vida, a recém-nascida evoluiu com apneia e crise convulsiva, permanecendo internada em Unidade de Terapia Intensiva Neonatal até um mês e vinte dias. Em tomografia computadorizada de crânio, realizada com quinze dias de vida, evidenciou-se hemisfério cerebelar de dimensões reduzidas associado a cistos em fossa posterior com comunicação com $4^{\circ}$ ventrículo, uma variante Dandy Walker. Em ultrassom abdominal, apresentava espessamento de parênquima renal bilateral.
A paciente foi admitida no Pronto Socorro Infantil com dois meses de idade, com padrão respiratório irregular associado à presença de hipotonia e de movimentos oculares anormais, o que caracterizou um exame neurológico anormal. No exame físico, apresentava taquipneia, com 80 respirações por minuto, ausculta pulmonar com murmúrio vesicular simétrico e ausência de sinais de desconforto respiratório. Na ausculta cardíaca e na propedêutica abdominal, não foram encontrados sopros cardíacos, visceromegalias ou massas palpáveis. Apresentava níveis pressóricos acima do percentil 95 para a idade (PA: $110 \times 70 \mathrm{mmHg}$ ), mensurados com manguito adequado ${ }^{(14)}$. No exame neurológico, notava-se a presença de movimentos oculares anormais, o perímetro cefálico era de $39 \mathrm{~cm}$ (percentil 50) e havia hipotonia generalizada. Não foram encontrados dismorfismos fenotípicos ou alterações músculo-esqueléticas.

Os exames laboratoriais iniciais evidenciavam alcalose respiratória, acidose metabólica e hipercalemia, com a seguinte gasometria venosa: $\mathrm{pH} 7,42, \mathrm{pCO}_{2} 13 \mathrm{mmHg}$, $\mathrm{HCO}_{3} 8 \mathrm{mEq} / \mathrm{L}$ e BE $15 \mathrm{mEq} / \mathrm{L}$. Os eletrólitos mostraram: $\mathrm{Na} 135 \mathrm{mEq} / \mathrm{L}, \mathrm{Ca} 9,2 \mathrm{mg} / \mathrm{dL}, \mathrm{K} 6,1 \mathrm{mg} / \mathrm{dL}, \mathrm{Mg} 1,6 \mathrm{mg} / \mathrm{dL}$, $\mathrm{Cl} 112 \mathrm{mEq} / \mathrm{L}$ e anion gap $15 \mathrm{mEq} / \mathrm{L}$ (normal:8-15).

Afastando-se as causas pulmonares e cardíacas que justificassem o referido quadro clínico, iniciou-se investigação neurológica por meio de exames de imagem, assim como a investigação de causas renais que explicassem a presença da acidose metabólica e da hipercalemia, que desapareceu durante a evolução clínica após controle da acidose metabólica. A investigação nefrológica mostrou os seguintes resultados laboratoriais e de imagem:

- Função renal normal: ureia $27 \mathrm{mg} / \mathrm{dL}$ e creatinina $0,5 \mathrm{mg} /$ dL; Urina I com pH 8, densidade 1010 leucócitos 12/ campo e eritrócitos $2 /$ campo.

- Ultrassom de rins e vias urinárias: rins aumentados de tamanho, com perda da diferenciação córtico-medular e aumento da ecogenicidade renal (rim direito: $7,5 \times 3,1 \times 3,0 \mathrm{~cm}$ e rim esquerdo: $7,2 \times 3,0 \times 4,7 \mathrm{~cm})$, com imagem de "favo de mel", sugerindo imagens císticas.

- Ressonância magnética de abdome: rins com múltiplos cistos em região cortical renal bilateral.

- Biópsia renal: presença de cistos tubulares com oxalato de cálcio e fibrose intersticial com glomérulos estruturalmente preservados.

- Teste de acidificação urinária: $\mathrm{pH} 5$, amônia $0,23 \mu \mathrm{Eq} / \mathrm{min}$; $\mathrm{HCO}_{3}<1 \mathrm{mmol} / \mathrm{L}$ e acidez titulável $26,2 \mu \mathrm{Eq} / \mathrm{min}$.

O teste de acidificação urinária deve ser realizado em vigência de acidose metabólica e tem por objetivo estudar o 
comprometimento tubular através da mensuração urinária do bicarbonato (avaliação do túbulo proximal) e das dosagens da amônia e acidez titulável (túbulo distal). Se o paciente não apresentar nível sérico de bicarbonato adequado para a realização do teste, habitualmente provoca-se a acidose através da administração de cloreto de amônia. No caso em questão, a paciente fez a prova basal, pois já se encontrava

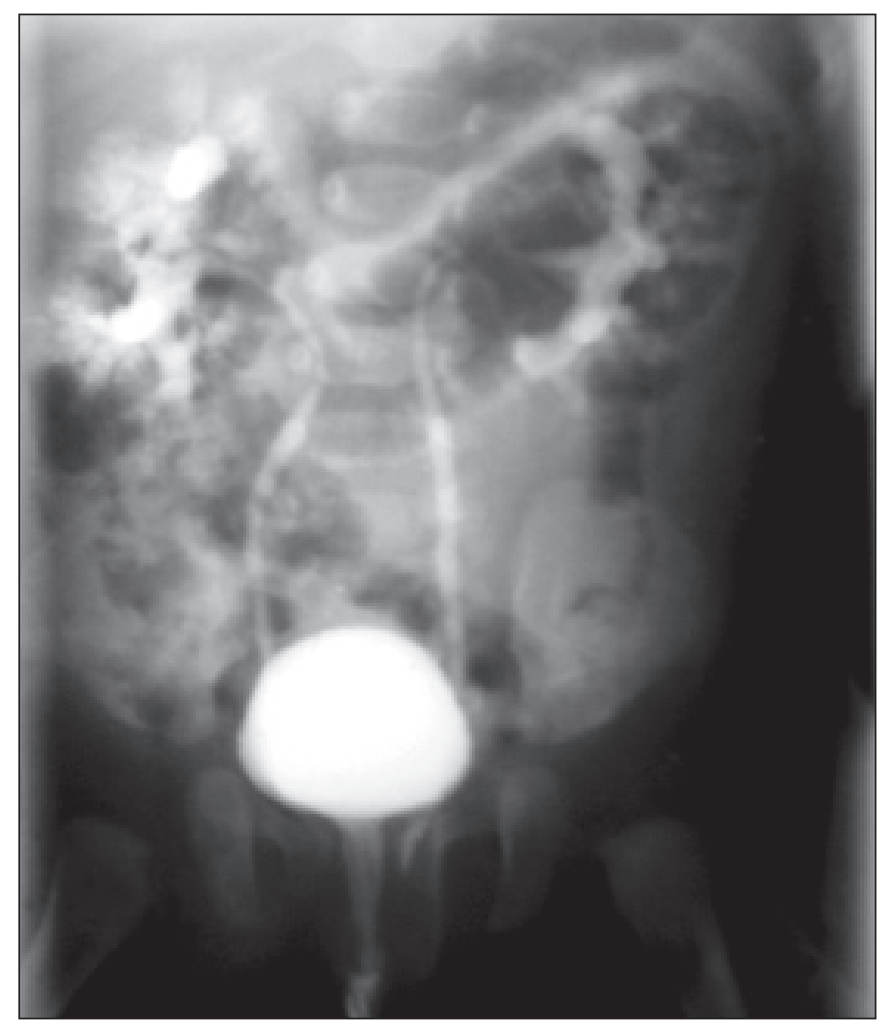

Figura 1 - Uretrocistografia miccional com evidência de refluxo vésico-ureteral grau $\mathrm{V}$ bilateral.

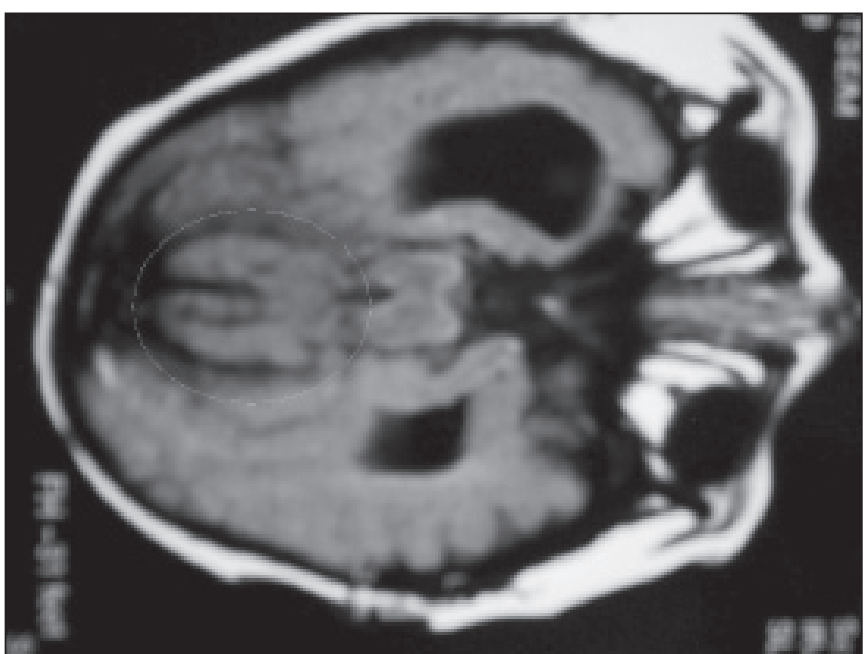

Figura 2 - Ressonância magnética de crânio com sinal do dente molar característico da Síndrome de Joubert. em acidose $\left(\mathrm{HCO}_{3}\right.$ no momento da prova de $8 \mathrm{mEq} / \mathrm{L}$ e BE de $-15 \mathrm{mEq} / \mathrm{L}$ ). Nesse caso, a interpretação do resultado foi feita com cuidado devido à baixa faixa etária da paciente (dois meses), uma vez que a função tubular só está totalmente desenvolvida aos seis meses de vida. Para melhor investigar as malformações renais observadas nos exames descritos, foi realizada uretrocistografia miccional que demonstrou presença de refluxo vesicoureteral grau V bilateral (Figura 1).

A investigação neurológica iniciou-se com a realização de tomografia de crânio, que mostrou agenesia de vérmis cerebelar e hidrocefalia compensatória. A ressonância magnética de crânio demonstrou hipoplasia de vérmis cerebelar e de corpo caloso, fossa interpeduncular profunda, proeminência de pedúnculo cerebelar - "sinal do dente molar" (figura semelhante a um dente molar, característica da síndrome de Joubert), como pode ser observado na Figura 2. Além disso, observou-se ventriculomegalia nesse exame de imagem. O exame de fundo de olho não evidenciou alterações.

A associação de ritmo respiratório irregular, comprometimento do desenvolvimento neuropsicomotor e malformações renais, bem como as alterações observadas nos exames de imagem sugeriram a hipótese diagnóstica de síndrome de Joubert.

Até os três meses de idade, a criança evoluiu com manutenção da hiperpneia e atraso do desenvolvimento, não apresentando deterioração da função renal e com normalização da pressão arterial, sem necessidade de drogas antihipertensivas.

\section{Comentários}

A síndrome de Joubert clássica é uma desordem autossômica recessiva caracterizada por hipoplasia de vérmis cerebelar, com pedúnculo cerebelar superior proeminente, o que leva ao "sinal do dente molar" em cortes axiais na ressonância magnética de crânio. Outros achados da síndrome de Joubert incluem: hipotonia, retardo de desenvolvimento neuropsicomotor, alterações oculares e ritmo respiratório irregular com hiperpneia/apneia ${ }^{(2-6)}$.

O nome síndrome de Joubert não se refere a uma entidade única, mas a um grupo de doenças heterogêneas do ponto de vista clínico e genético. Mutações em pelo menos sete genes diferentes podem estar associadas aos diferentes tipos da síndrome: o gene AHI1 em 6q23, NPHP1 em 2q13, CEP290 (NPHP6) em 12q21.32, TMEM67 em 8q21, RPGRIP1L em 16q12.2, e dois outros genes em 9q34 e 11p12-q13.

As desordens relacionadas à síndrome de Joubert (Tabela 1) consideradas variantes da forma clássica ${ }^{(15-20)}$ associam- 
se a variações no quadro clínico clássico, tais como: outras anormalidades do sistema nervoso central, polidactilia, coloboma ocular, distrofia retiniana, doença renal incluindo displasia cística e nefronoftise ${ }^{(21-24)}$. Na Tabela 2, são descritas as variantes mais comuns.

A doença renal é descrita em $30 \%$ dos indivíduos acometidos pela síndrome de Joubert. As alterações renais podem ser divididas em dois subtipos: displasia cística e nefronoftise juvenil ${ }^{(1)}$. A displasia cística é caracterizada pela presença de múltiplos cistos de dimensões variáveis, visualizados em ultrassom de rins e vias urinárias e podem estar presentes desde o nascimento. O achado de displasia cística é característico da variante da síndrome de Joubert conhecida como Dekaban-Arima ${ }^{(24-26)}$.

A nefronoftise juvenil é caracterizada por microcistos em túbulos renais, causando defeitos na concentração urinária. Manifesta-se com polidipsia, poliúria, anemia, déficit de crescimento, podendo evoluir para doença renal terminal. No curso da doença, é possível observar diminuição das dimensões renais, bem como presença de cicatrizes em parênquima renal por meio de exame de ultrassonografia ${ }^{(27)}$.

Essa paciente apresentou os achados característicos da síndrome de Joubert, que compreendem o sinal do dente molar, as alterações do padrão respiratório e do desenvolvimento neurológico, além das malformações renais. O que diferencia esse caso dos previamente descritos é a presença de refluxo
Tabela 1 - Variantes clínicas da Síndrome de Joubert

\begin{tabular}{|c|c|c|}
\hline Genética & Frequência & $\begin{array}{l}\text { Variante do } \\
\text { quadro clínico }\end{array}$ \\
\hline $\begin{array}{l}\text { Deleção } \\
\text { homozigota gene } \\
\text { NPHP1 }\end{array}$ & 1 a $2 \%$ & $\begin{array}{l}\text { Encefalocele, } \\
\text { meningomielocele, } \\
\text { polimicrogiria, } \\
\text { distrofia retiniana, } \\
\text { nefronoftise juvenil, } \\
\text { doença cística } \\
\text { medular renal }\end{array}$ \\
\hline $\begin{array}{l}\text { Mutação gene } \\
\text { AHI1 }\end{array}$ & 10 a $15 \%$ & $\begin{array}{l}\text { Polimicrogiria, } \\
\text { distrofia retiniana, } \\
\text { nefronoftise }\end{array}$ \\
\hline $\begin{array}{l}\text { Mutação gene } \\
\text { CEP290 }\end{array}$ & $10 \%$ & $\begin{array}{l}\text { Encefalocele, } \\
\text { distrofia retiniana, } \\
\text { coloboma ocular, } \\
\text { amaurose } \\
\text { congênita, } \\
\text { nefronoftise }\end{array}$ \\
\hline
\end{tabular}

vesicoureteral, além dos rins de tamanho aumentado para a faixa etária. O exame anátomo-patológico mostrou a presença de cistos tubulares com fibrose intersticial em fragmento córtico-medular renal, porém, devido ao fato de o material ser pouco representativo (5 glomérulos), não foi possível definir o quadro como nefronoftise. É possível que se possa, durante a evolução da doença, definir de forma precisa o tipo

Tabela 2 - Manifestações clínicas das variantes da Síndrome de Joubert

\begin{tabular}{|c|c|c|c|c|}
\hline Manifestações clínicas & $\begin{array}{c}\text { Síndrome } \\
\text { Joubert }\end{array}$ & $\begin{array}{c}\text { Síndrome } \\
\text { Dekaban-Arima }\end{array}$ & $\mathrm{COACH}$ & Nefronoftise juvenil \\
\hline \multicolumn{5}{|l|}{ SNC } \\
\hline Sinal do dente molar & + & $(+)$ & $(+)$ & $(+)$ \\
\hline Outras anormalidades de SNC & $(+)$ & ND & ND & - \\
\hline Retardo DNPM & + & + & + & $(+)$ \\
\hline Hipotonia & + & + & + & $(+)$ \\
\hline Polimicrogiria & $(+)$ & ND & ND & ND \\
\hline \multicolumn{5}{|l|}{ Oftalmológica } \\
\hline Apraxia oculomotora & $(+)$ & $(+)$ & $(+)$ & $(+)$ \\
\hline Distrofia retiniana & $(+)$ & + & ND & - \\
\hline Coloboma & - & $(+)$ & + & - \\
\hline Amaurose congenita & $(+)$ & + & $(+)$ & $(+)$ \\
\hline \multicolumn{5}{|l|}{ Renal } \\
\hline Displasia cística renal & $(+)$ & + & - & - \\
\hline Nefronoftise & $(+)$ & - & $(+)$ & + \\
\hline \multirow[t]{3}{*}{ Genes } & NPHP1 & ND & ND & NPHP1 \\
\hline & $\mathrm{AHI} 1$ & & & CEP 290 \\
\hline & CEP 290 & & & \\
\hline
\end{tabular}

$\mathrm{COACH}$ : hipoplasia de vérmis cerebelar, oligofrenia, ataxia, colobomas, fibrose hepática + presente; - ausente; (+) pode estar presente; ND: não descrito na literatura; DNPM: desenvolvimento neuropsicomotor 
de comprometimento renal que a paciente apresenta, visto que na nefronoftise familiar juvenil, em geral, há surgimento de poliúria, polidpsia, anemia e atraso de crescimento antes do aparecimento da insuficiência renal terminal. Nos casos de nefronoftise, a avaliação genética por meio da extensão da deleção [NPHP1, DEL] demonstra não haver diferença entre os pacientes com fenótipo renal isolado e aqueles com sintomas neurológicos associados ${ }^{(28)}$.

\section{Referências bibliográficas}

1. Parisi MA, Doherty D, Chance PF, Glass IA. Joubert syndrome (and related disorders). Eur J Hum Genet 2007;15:511-21.

2. Gleeson JG, Keeler LC, Parisi MA, Marsh SE, Chance PF, Glass IA et al. Molar tooth sign of the midbrain-hindbrain junction: occurrence in multiple distinct syndromes. Am J Med Genet 2004;125A:125-34.

3. Saraiva JM, Baraitser M. Joubert syndrome: a review. Am J Med Genet 1992:43:726-31.

4. Sztriha L, Al-Gazali LI, Aithala GR, Nork M. Joubert's syndrome: new cases and review of clinicopathologic correlation. Pediatr Neurol 1999;20: 274-81.

5. Holroyd S, Reiss AL, Bryan RN. Autistic features in Joubert syndrome: a genetic disorder with agenesis of the cerebellar vermis. Biol Psychiatry 1991;29:287-94.

6. Hodgkins PR, Harris CM, Shawkat FS, Thompson DA, Chong K, Timms $\mathrm{C}$ et al. Joubert syndrome: long-term follow-up. Dev Med Child Neurol 2004;46:694-9.

7. Steinlin M, Schmid M, Landau K, Boltshauser E. Follow-up in children with Joubert syndrome. Neuropediatrics 1997;28:204-11.

8. Raynes HR, Shanske A, Goldberg S, Burde R, Rapin I. Joubert syndrome: monozygotic twins with discordant phenotypes. J Child Neurol 1999;14: 649-54.

9. Maria BL, Boltshauser E, Palmer SC, Tran TX. Clinical features and revised diagnostic criteria in Joubert syndrome. J Child Neurol 1999;14: 583-90.

10. Assadi F. Lack of NPHP2 mutations in a newborn infant with Joubert syndromerelated disorder presenting as end-stage renal disease. Pediatr Nephrol 2007;22:750-2.

11. Dekaban AS. Hereditary syndrome of congenital retinal blindness (Leber), polycystic kidneys and maldevelopment of the brain. Am J Ophthalmol 1969;68:1029-37.

12. Quisling RG, Barkovich AJ, Maria BL. Magnetic resonance imaging features and classification of central nervous system malformations in Joubert syndrome. J Child Neurol 1999;14:628-35.

13. Parisi MA, Doherty D, Eckert ML, Shaw DW, Ozyurek H, Aysun S et al. AHI1 mutations cause both retinal dystrophy and renal cystic disease in Joubert syndrome. J Med Genet 2006;43:334-9.

14. Brewer ED. Evaluation of hypertension in childhood diseases. In: Avner ED, Harmon WE, Niaudet P, editors. Pediatric nephrology. 5th ed. Philadelphia: Lippincott Williams \& Wilkins; 2004. p. 1179-97.
Pode-se concluir que a Síndrome de Joubert é uma doença autossômica recessiva multissistêmica, caracterizada por aplasia do vermis cerebelar, retardo mental, hipotonia muscular e padrão respiratório irregular, associados a movimentos oculares anormais. Alguns pacientes podem evoluir para doença renal terminal, indicando-se, assim, investigação de malformações renais e avaliação da função renal sempre que houver suspeita da síndrome.

15. Valente EM, Brancati F, Silhavy JL, Castori M, Marsh SE, Barrano G et al. AHI1 gene mutations cause specific forms of Joubert syndrome-related disorders. Ann Neurol 2006;59:527-34.

16. Utsch B, Sayer JA, Attanasio M, Pereira RR, Eccles M, Hennies HC et al Identification of the first AHI1 gene mutations in nephronophthisis-associated Joubert syndrome. Pediatr Nephrol 2006;21:32-5.

17. Ferland RJ, Eyaid W, Collura RV, Tully LD, Hill RS, Al-Nouri D et al. Abnormal cerebellar development and axonal decussation due to mutations in AHI1 in Joubert syndrome. Nat Genet 2004;36:1008-13.

18. Castori M, Valente EM, Donati MA, Salvi S, Fazzi E, Procópio E et al. NPHP1 gene deletion is a rare cause of Joubert syndrome related disorders. $\mathrm{J}$ Med Genet 2005;42:e9.

19. Hildebrant F, Nothwang HG, Vossmerbäumer U, Springer C, Strahm B, Hoppe $B$ et al. Lack of large, homozygous deletions of the nephronophthisis 1 region in Joubert syndrome type B. Pediatr Nephrol 1998;12:16-9.

20. Saunier S, Salomon R, Antignac C. Nephronophthisis. Curr Opin Genet Dev 2005;15:324-31.

21. Doherty D, Glass IA, Siebert JR, Parisi MA, Shaw DW, Chance PF et al Prenatal diagnosis in pregnancies at risk for Joubert syndrome by ultrasound and MRI. Prenat Diagn 2005;25:442-7.

22. Aslan H, Ulker V, Gulcan EM, Numanoglu C, Gul A, Agar M et al. Prenatal diagnosis of Joubert syndrome: a case report. Prenat Diagn 2002;22:13-6.

23. Fluss J, Blaser S, Chitayat D, Akoury H, Glanc P, Skidmore M et al. Molar tooth sign in fetal brain magnetic resonance imaging leading to the prenatal diagnosis of Joubert syndrome and related disorders. J Child Neurol 2006;21:320-4.

24. Kumada S, Hayashi M, Arima K, Nakayama H, Sugai K, Sasaki M et al. Renal disease in Arima syndrome is nephronophthisis as in other Joubert-related Cerebello-oculo-renal syndromes. Am J Med Genet A 2004;131:71-6.

25. Hildebrandt F, Rensing C, Betz R, Sommer U, Birnbaun S, Imm A et al. Establishing an algorithm for molecular genetic diagnostics in 127 families with juvenile nephronophthisis. Kidney Int 2001;59:434-45.

26. Valente EM, Marsh SE, Castori M, Dixon-Salazar T, Bertini E, Al-Gazali L et al Distinguishing the four genetic causes of jouberts syndrome-related disorders. Ann Neurol 2005;57:513-9.

27. Caridi G, Dagnino M, Rossi A, Valente EM, Bertini E, Fazzi E et al. Nephronophthisis type 1 deletion syndrome with neurological symptoms: prevalence and significance of the association. Kidney Int. 2006;70:1342-7.

28. Hildebrandt F, Waldherr R, Kutt R, Brandis $M$. The nephronophthisis complex: clinical and genetic aspects. Clin Investig 1992;70:802-8. 